\title{
Options for Assessing Joints and Entheses in Psoriatic Arthritis by Ultrasonography and Magnetic Resonance Imaging: How to Move Forward
}

\author{
Lihi Eder, Sibel Z. Aydin, Gurjit S. Kaeley, Walter P. Maksymowych, and Mikkel Østergaard
}

\begin{abstract}
Psoriatic arthritis (PsA) is a heterogeneous disease with various manifestations of musculoskeletal inflammation. Recent advances in imaging, including ultrasound (US) and magnetic resonance imaging (MRI), allow for the accurate evaluation of the extent of inflammation and damage in the peripheral joints, spine, and entheses. The development and validation of outcome measures are critical steps in creating standardized evaluations of musculoskeletal inflammation and damage in psoriatic patients. At the 2017 meeting of the Group for Research and Assessment of Psoriasis and PsA (GRAPPA), recent work on outcome measures from the GRAPPA US and MRI working groups was summarized. The GRAPPA US group has been developing and validating a sonographic enthesitis scoring system in PsA. The GRAPPA MRI group focuses on the evaluation of whole-body MRI for the assessment of musculoskeletal inflammation in the joints and entheses in patients with PsA. (J Rheumatol Suppl. 2018 June;94:44-7; doi:10.3899/jrheum.180140)
\end{abstract}

Key Indexing Terms:

PSORIASIS

GRAPPA

$\begin{array}{cr}\text { PSORIATIC ARTHRITIS } & \text { JOINTS } \\ \text { ULTRASOUND } & \text { MAGNETIC RESONANCE IMAGING }\end{array}$

Psoriatic arthritis (PsA) is a heterogeneous disease with various manifestations of musculoskeletal inflammation. Advances in imaging, including ultrasound (US) and magnetic resonance imaging (MRI), have allowed for the accurate evaluation of the extent of inflammation and damage in the peripheral joints, spine, and entheses. The development and validation of outcome measures is a critical step in allowing for a standardized evaluation of musculoskeletal inflammation and damage in psoriatic patients. The following is a summary of recent outcome measure work from the

From the University of Toronto and Women's College Hospital, Toronto; University of Ottawa, Ottawa Hospital Research Institute, Ottawa, Ontario; University of Alberta, Edmonton, Alberta, Canada; University of Florida College of Medicine, Jacksonville, Florida, USA; Copenhagen Center for Arthritis Research, Center for Rheumatology and Spine Diseases, Rigshospitalet, Glostrup; Department of Clinical Medicine, Faculty of Health and Medical Sciences, University of Copenhagen, Copenhagen, Denmark.

As part of the supplement series GRAPPA 2017, this report was reviewed internally and approved by the Guest Editors for integrity, accuracy, and consistency with scientific and ethical standards.

L. Eder, MD, PhD, Assistant Professor of Medicine, University of Toronto and Women's College Hospital; S.Z. Aydin, MD, Associate Professor of Medicine, University of Ottawa, Ottawa Hospital Research Institute; G.S. Kaeley, MD, MBBS, MRCP, RhMSUS, Professor of Medicine, University of Florida College of Medicine; W.P. Maksymowych, MB, ChB, FRCPC, Professor of Medicine, University of Alberta; M. Østergaard, MD, PhD, Professor of Medicine, Copenhagen Center for Arthritis Research, Center for Rheumatology and Spine Diseases, Rigshospitalet, and Department of Clinical Medicine, Faculty of Health and Medical Sciences, University of Copenhagen.

Address correspondence to Dr. L. Eder, Women's College Hospital,

76 Grenville St., Toronto, Ontario M5S 1B2, Canada.

E-mail:lihi.eder@wchospital.ca
Group for Research and Assessment of Psoriasis and Psoriatic Arthritis (GRAPPA) US and MRI working groups as presented at the GRAPPA 2017 annual meeting in Amsterdam, the Netherlands.

\section{US Working Group: Assessment of Sonographic Enthesitis in PsA}

Enthesitis assessment is recommended in every patient with $\mathrm{PsA}^{1}$. However, the accuracy of clinical examination of enthesitis is limited because it relies primarily on subjective findings of tenderness over entheseal sites. US imaging can show entheses in high fidelity, thus improving the accuracy of enthesitis diagnoses in patients with $\mathrm{PsA}^{2}$.

In 2014, the Outcome Measures in Rheumatology (OMERACT) US special interest group reached a consensus regarding the sonographic elementary lesions defining spondyloarthritis (SpA)-related enthesitis ${ }^{3}$. This was an important first step toward ensuring a high degree of consistency across studies. However, while this group defined the concept of enthesitis at the level of the enthesis, it did not address how to score the degree or severity of enthesitis activity at the global patient level.

Numerous studies have used US to evaluate enthesitis at the global patient level in PsA to study aspects of the disease, such as early diagnosis and improved care ${ }^{4}$. Although several global sonographic enthesitis scoring systems have been developed, many studies used variations of these methods or other nonvalidated scoring systems. This lack of standardization has limited the ability to compare results across studies. In addition, very few studies controlled for important

Personal non-commercial use only. The Journal of Rheumatology Copyright $@$ 2018. All rights reserved 
confounding factors in PsA, such as obesity. Therefore, the GRAPPA US working group concentrated on the sonographic assessment of enthesitis in PsA as its initial project.

Systematic literature review of sonographic enthesitis scores. The first step in this process included a systematic literature review (SLR) to evaluate the current evidence and knowledge gaps in scoring systems of enthesitis in PsA ${ }^{5}$. The search strategy was constructed to find original publications containing terms related to US, enthesitis, SpA, or PsA. Data extraction focused on the enthesitis scoring system properties used in each study and followed each OMERACT filter component: reliability, feasibility, discriminative validity, responsiveness, and construct validity as they related to clinical enthesitis assessment, inflammation biomarkers, and enthesitis imaging by other modalities. The SLR included a total of 51 of 310 identified manuscripts. Only 1 scoring system was developed and validated in patients with PsA. Only $18(35 \%)$ of the studies included patients with PsA, while the rest focused on axial SpA. Construct validity of enthesitis scores was assessed using biomarkers, clinical examination, and imaging (MRI and radiographs) in only 1 (2\%), $11(21.5 \%)$, and 0 studies in patients with PsA, respectively. Responsiveness was assessed in 7 studies, none of which included patients with PsA. Only 2 (4\%) studies assessed discriminative validity in PsA, and only 1 study evaluated the effect of body mass index and age on sonographic enthesitis. Overall, the results of the SLR highlight existing gaps in knowledge regarding the validity of existing enthesitis sonographic scoring systems in PsA.

Preliminary enthesitis score in PsA. Because of these notable limitations, the GRAPPA US working group set a goal to develop and validate a novel enthesitis sonographic score for PsA through a combined data-driven and expert-opinion driven approach. It then included a pilot study to collect preliminary data regarding the performance of various sonographic entheseal lesions and sites in distinguishing between PsA and healthy controls that will inform the development of a novel sonographic enthesitis score for PsA. In this pilot study, a total of 100 age- and sex-matched individuals (50 PsA and 50 controls) were evaluated ${ }^{6}$. Eleven entheseal sites were scanned bilaterally according to a standardized protocol. Because the OMERACT definition for elementary lesions for enthesitis does not include a scoring system, each elementary lesion was scored 1 point for its presence. A series of regression models was used to find the optimal combination of entheseal sites and elementary lesions that distinguished PsA from controls. The optimal model included 5 elementary lesions (enthesophytes, Doppler signal, erosions, thickness, and structural changes) and 6 entheseal sites (proximal and distal patellar ligament attachments, Achilles tendon, plantar fascia, lateral epicondyle, and supraspinatus). The area under the receiver-operation characteristic curve for this model was 0.93 (95\% CI 0.88-0.98). This study identified potential elementary lesions and entheseal sites that could distinguish PsA and controls with high accuracy. This information will contribute to the development of a new sonographic enthesitis scoring system in patients with PsA. The GRAPPA US group aims to further develop and validate the new scoring system using an independent cohort of patients with PsA and controls, as well as to evaluate the scoring system's discriminative ability and responsiveness.

\section{MRI Working Group}

PsA is a heterogeneous disease that involves both peripheral and axial joints and entheses. MRI is the only imaging modality that allows assessment of all inflammatory components [synovitis, tenosynovitis, osteitis, enthesitis, periarticular inflammation, and bone marrow edema (BME)], as well as structural damage (bone erosion and bone proliferation) in the peripheral joints and entheses of patients with PsA. Further, MRI can also assess inflammation and damage in the joints and entheses of the axial skeleton, i.e., the spine, sacroiliac joints, and anterior chest wall.

Unfortunately, the widespread and variable PsA disease manifestations have challenged the development of MRI outcome measures that identify the disease activity in all patients with PsA because conventional MRI methods have allowed only for the assessment of a selected area, e.g., a hand, knee, or entheseal region. However, recent technical developments have made MRI of the entire body in 1 imaging session (whole-body MRI; WB-MRI) possible. The following is a discussion of conventional and new MRI techniques and a proposal for an MRI approach that may bring crucial new knowledge regarding the optimal use of MRI as an outcome measure for monitoring disease activity and damage in PsA.

Approaches to MRI assessment of 1 or few joints. Semiquantitative scoring systems for synovitis, BME, and/or erosions in peripheral PsA have been described, but most of these have only been used in a few patients. The international MRI in arthritis group of OMERACT has developed the Psoriatic Arthritis Magnetic Resonance Imaging MRI Score (PsAMRIS) for evaluation of inflammatory (synovitis, tenosynovitis, periarticular inflammation, osteitis) and destructive (bone erosion, new bone formation) changes in PsA hands and feet ${ }^{7}$. PsAMRIS is the only validated assessment system available in PsA and has a documented good intra- and interreader reliability for status and change scores of all variables. In addition, the inflammatory variables have also demonstrated good sensitivity to change and discrimination ${ }^{7,8,9}$. The OMERACT PsAMRIS is currently the method of choice for MRI assessment of patients with PsA in clinical trials.

Quantitative methods. Quantitative methods previously applied in PsA include quantification of contrast enhancement by dynamic contrast-enhanced MRI ${ }^{10}$. Further, different methods of quantification of synovitis, BME, and

Personal non-commercial use only. The Journal of Rheumatology Copyright (C) 2018. All rights reserved. 
bone erosions have been described in other inflammatory arthritides 8,11 and in osteoarthritis (OA) of the knee and hip $^{12,13,14,15,16}$. The latter, the Knee and Hip Inflammation Magnetic Resonance Imaging Scoring Systems (KIMRISS and HIMRISS, respectively), are planned to be applied in the proposed development plan and will, therefore, be described further.

KIMRISS and HIMRISS focus on the assessment of active lesions in the hip and knee, specifically, bone marrow lesions (BML) and synovitis effusion ${ }^{12,13,14,15,16}$. Although originally validated in OA, these scoring methods can, in principle, be applied to the objective assessment of hip and knee inflammation in other inflammatory joint disorders such as PsA. The principal innovation of these imaging scoring tools lies in their application of electronic overlays on a Web-based interface to facilitate rapid touch- or click-based binary scoring of BML in many small regions of bone on fluid-sensitive (intermediate-weighted fat-saturated or short-tau inversion recovery) sequences (see www.carearthritis.com, "Osteoarthritis Imaging" under Imaging Portal). The presence or absence of BML is scored on a dichotomous basis on consecutive slices through the joint rather than according to the percent volume of a bone region that contains BML.

In KIMRISS, BML is scored using specific overlays for the femur, tibia, and patella on consecutive sagittal slices through the knee joint. KIMRISS has been shown to have very good reliability for status and change scores in a patient sample $(n=80)$ from the Osteoarthritis Initiative observational cohort that was assessed over a year ${ }^{15,16}$. Responsiveness to change and correlation with Western Ontario and McMaster Universities Osteoarthritis Index scores for knee pain were also demonstrable in a pilot evaluation of adalimumab over 12 weeks in patients with inflammatory knee $\mathrm{OA}^{16}$.

In HIMRISS, BML is scored using a single overlay positioned over the femoral head and extending into the acetabulum on consecutive coronal slices through the hip joint ${ }^{13}$. HIMRISS has been shown to have very good to excellent reliability for status and change scores, especially in the femoral head, in an observational study of 40 patients where scans were obtained 8 weeks before and after steroid injection into the hip joint ${ }^{13}$. Standardized calibration modules based on a Web-based interface and real-time iterative feedback referenced to expert reader scores for individual bone regions have been developed and validated for each method to facilitate the attainment of prespecified acceptable targets for reliability ${ }^{14,15}$.

Approaches to MRI assessment of many joints (WB-MRI). WB-MRI is a relatively new technique that allows for the assessment of the entire body in 1 examination in less than an hour. WB-MRI can potentially provide a global assessment of the inflammatory status of a patient with arthritis ${ }^{17,18,19,20}$. This may improve the utility of MRI in ankylosing spondylitis, rheumatoid arthritis, and particularly PsA, which presents with varying patterns of arthritis, enthe- sitis, spondylitis, and/or dactylitis. The OMERACT MRI in arthritis group has taken the first steps toward standardizing WB-MRI image acquisition and assessment, as well as an OMERACT scoring system ${ }^{19}$. After an SLR, the MRI working group decided to primarily focus on inflammation in peripheral joints and entheses. It then developed MRI definitions for these pathologies, selected anatomical locations for assessment, agreed on a core set of MRI sequences and imaging planes for the different regions, and proposed a preliminary semiquantitative scoring system. In addition, it was decided to test and further develop the system in ongoing iterative multireader exercises ${ }^{19}$. A Web-based case report form with line drawings has been developed to facilitate correct registration of bone and soft tissue inflammation in all peripheral joints and entheses.

How to move forward. The GRAPPA MRI working group proposes to perform a longitudinal, multicenter, and preferably randomized treatment study of patients with active PsA, which will build on WB-MRI assessment of peripheral and axial joints and entheses according to the OMERACT recommendations. This study would also incorporate assessments of hands and feet according to PsAMRIS and knees and hips according to KIMRISS/HIMRISS. This approach will allow both the objective assessment of the therapeutic effect of the tested drug by validated MRI methods as well as simultaneously generate MRI data that will provide crucial information about the relative performance of different MRI approaches and their best combinations regarding assessment of patients with PsA in clinical trials and practice.

The varying pattern of involvement of axial and peripheral joints and entheses challenges the use of MRI and US in PsA clinical trials and observational studies. US is an accessible and cost-effective modality for enthesitis assessment; however, the development and validation of clinical outcome measures for enthesitis evaluation is an unmet need. WB-MRI allows for the evaluation of the various features of musculoskeletal inflammation in PsA and is extremely promising for the objective assessment of inflammation in PsA. The technical and methodological development and validation of different WB-MRI assessment methods, in combination with more detailed assessments of selected areas, such as hips and knees, are highly relevant.

\section{REFERENCES}

1. Coates LC, Kavanaugh A, Mease PJ, Soriano ER, Laura Acosta-Felquer M, Armstrong AW, et al. Group for Research and Assessment of Psoriasis and Psoriatic Arthritis 2015 treatment recommendations for psoriatic arthritis. Arthritis Rheumatol 2016;68:1060-71.

2. D'Agostino MA. Ultrasound imaging in spondyloarthropathies. Best Pract Res Clin Rheumatol 2010;24:693-700.

3. Terslev L, Naredo E, Iagnocco A, Balint PV, Wakefield RJ, Aegerter $\mathrm{P}$, et al; Outcome Measures in Rheumatology Ultrasound Task Force. Defining enthesitis in spondyloarthritis by ultrasound: results of a Delphi process and of a reliability reading exercise. Arthritis Care Res 2014;66:741-8.

Personal non-commercial use only. The Journal of Rheumatology Copyright @ 2018 . All rights reserved. 
4. Zabotti A, Bandinelli F, Batticciotto A, Scire CA, Iagnocco A, Sakellariou G; Musculoskeletal Ultrasound Study Group of the Italian Society of Rheumatology. Musculoskeletal ultrasonography for psoriatic arthritis and psoriasis patients: a systematic literature review. Rheumatology 2017;56:1518-32.

5. Elalouf O, Bakirci S, Touma Z, Anderson M, Kaeley G, Aydin S, et al. Psoriatic arthritis sonographic enthesitis scores - systematic review of the literature [abstract]. Arthritis Rheumatol 2017;69 Suppl 10:254.

6. Tom S, Zhong Y, Cook R, Aydin S, Kaeley G, Eder L. Development of an ultrasonographic enthesitis score for psoriatic arthritis patients [abstract]. Arthritis Rheumatol 2017;69 Suppl 10:1874.

7. Ostergaard M, McQueen F, Wiell C, Bird P, Bøyesen P, Ejbjerg B, et al. The OMERACT psoriatic arthritis magnetic resonance imaging scoring system (PsAMRIS): definitions of key pathologies, suggested MRI sequences, and preliminary scoring system for PsA Hands. J Rheumatol 2009;36:1816-24.

8. Bøyesen P, McQueen FM, Gandjbakhch F, Lillegraven S, Coates L, Wiell C, et al. The OMERACT Psoriatic Arthritis Magnetic Resonance Imaging Score (PsAMRIS) is reliable and sensitive to change: results from an OMERACT workshop. J Rheumatol 2011;38:2034-8.

9. Glinatsi D, Bird P, Gandjbakhch F, Mease PJ, Bøyesen P, Peterfy CG, et al. Validation of the OMERACT Psoriatic Arthritis Magnetic Resonance Imaging Score (PsAMRIS) for the hand and foot in a randomized placebo-controlled trial. J Rheumatol 2015;42:2473-9.

10. Cimmino MA, Parodi M, Innocenti S, Succio G, Banderali S, Silvestri E, et al. Dynamic magnetic resonance of the wrist in psoriatic arthritis reveals imaging patterns similar to those of rheumatoid arthritis. Arthritis Res Ther 2005;7:R725-31.

11. Ostergaard M. Magnetic resonance imaging in rheumatoid arthritis. Quantitative methods for assessment of the inflammatory process in peripheral joints. Dan Med Bull 1999;46:313-44.

12. Maksymowych WP, Cibere J, Loeuille D, Weber U, Zubler V, Roemer FW, et al. Preliminary validation of 2 magnetic resonance image scoring systems for osteoarthritis of the hip according to the OMERACT filter. J Rheumatol 2014;41:370-8

13. Maksymowych WP, Pitts M, Budak MJ, Gracey D, Lambert RG, McDougall D, et al. Development and preliminary validation of a digital overlay-based learning module for semiquantitative evaluation of magnetic resonance imaging lesions in osteoarthritis of the hip. J Rheumatol 2016;43:232-8.

14. Jaremko JL, Azmat O, Lambert RGW, Bird P, Haugen IK, Jans L, et al. Validation of a knowledge transfer tool according to the OMERACT filter: does web-based real-time iterative calibration enhance the evaluation of bone marrow lesions in hip osteoarthritis? J Rheumatol 2017:44:1713-7.

15. Jaremko JL, Azmat O, Lambert RG, Bird P, Haugen IK, Jans L, et al. Validation of a knowledge transfer tool for the knee inflammation MRI scoring system for bone marrow lesions according to the OMERACT filter: data from the Osteoarthritis Initiative. J Rheumatol 2017;44:1718-22.

16. Jaremko JL, Jeffery D, Buller M, Wichuk S, McDougall D, Lambert RG, et al. Preliminary validation of the Knee Inflammation MRI Scoring System (KIMRISS) for grading bone marrow lesions in osteoarthritis of the knee: data from the Osteoarthritis Initiative. RMD Open 2017;3:e000355.

17. Weckbach S, Schewe S, Michaely HJ, Steffinger D, Reiser MF, Glaser C. Whole-body MR imaging in psoriatic arthritis: additional value for therapeutic decision making. Eur J Radiol 2011;77:149-55.

18. Poggenborg RP, Pedersen SJ, Eshed I, Sørensen IJ, Møller JM, Madsen OR, et al. Head-to-toe whole-body MRI in psoriatic arthritis, axial spondyloarthritis and healthy subjects: first steps towards global inflammation and damage scores of peripheral and axial joints. Rheumatology 2015;54:1039-49.

19. Østergaard M, Eshed I, Althoff CE, Poggenborg RP, Diekhoff T, Krabbe S, et al. Whole-body magnetic resonance imaging in inflammatory arthritis: systematic literature review and first steps toward standardization and an OMERACT Scoring System. J Rheumatol 2017;44:1699-705.

20. Krabbe S, Ostergaard M, Eshed I, Sorensen I, Jensen B, Moller J, et al. Whole-body magnetic resonance imaging in axial spondyloarthritis: reduction of sacroiliac, spinal, and entheseal inflammation in a placebo-controlled trial of adalimumab. J Rheumatol 2018 (In press). 